\title{
COR-65 (Confusión, Oxigenación SaO2/FiO2 $\leq 300$, FR, PA) como Puntaje Alternativo para la Predicción de Complicaciones en Neumonía.
}

\section{Alirio Rodrigo Bastidas Goyes, Bastidas Goyes Alirio, Sharon Lechtig, Oscar Arcos, Victor Ramirez, Daniela Granados.}

\section{INTRODUCCIÓN}

La medición de la oxigenación en pacientes con neumonía está integrada en los puntajes pronósticos de PSI/PORT, SCAP, SOAR, ATS, IDSA y SMART-COP, sin embargo, no se conoce el rendimiento de un puntaje que integre valores de saturación arterial de oxígeno/fracción inspirada de oxígeno (SaO2/FiO2) para desenlaces de mortalidad y ventilación mecánica (VM) en estos pacientes.

\section{OBEJETIVO}

Determinar la validez pronostica de un puntaje que integre la relación SaO2/FiO2 en neumonía.

\section{MATERIAL Y MEDOTOS}

Estudio de cohorte retrospectiva, en sujetos mayores de 18 años que se ingresaron al servicio de urgencias y hospitalización de dos centros de atención en salud con diagnóstico de neumonía adquirida en la comunidad, se calculó el puntaje de CURB-65, CRB-65 al ingreso, y toma de gases arteriales, neumonía se definió como un cuadro de infección del parénquima pulmonar aguda, al menos un signo de respuesta inflamatoria sistémica, asociada con una opacidad nueva en la radiografía de tórax. Se evalúo posteriormente el rendimiento pronóstico de la $\mathrm{SaO} 2$ y de la relación $\mathrm{SaO} 2 / \mathrm{FiO} 2$ de ingreso con fallecimiento y requerimiento de VM, posteriormente con un punto de corte $\leq 300$ de $\mathrm{SaO} 2 / \mathrm{FiO} 2$ se evaluó un puntaje compuesto con esta variable y las variables habituales del CURB.

\section{RESULTADOS}

Ingresaron al análisis 487 sujetos, el 6,4\% falleció, 18,5\% requirió VM, 18,3\% con ingreso a UCI, el promedio de edad fue de 69,8(DE:20,8) años, 103(62,2\%) de sexo masculino, el promedio de FR fue: 22,1(DE:4,7), PAS:123,0(DE:23,5), PAD: 71,7(DE:14,5), BUN: 26,0(DE:17,0), SaO2:88,1(DE:6,8), para muerte el CURB-65 mostró un ACOR de: 0,685(IC95\%:0,594-0,777)( $p=0,001)$, CRB-65 ACOR: 0,641(IC95\%:0,534-0,749)(p=0,008), SaO2 ACOR: 0,479(IC95\%:0,369-0,589)( $\mathrm{p}=0,694)$, SaO2/FiO2 ACOR:

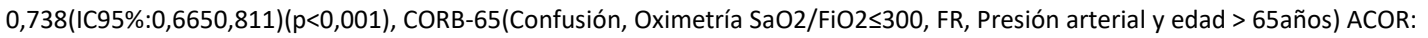

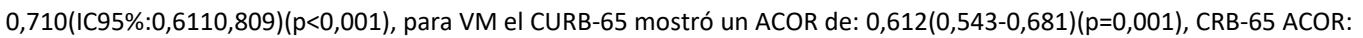
0,589(IC95\%:0,5180,659)(p=0,008), SaO2 ACOR: 0,468(IC95\%:0,401-0,535)(p=0,338), SaO2/FiO2 ACOR: 0,830(IC95\%:0,778-

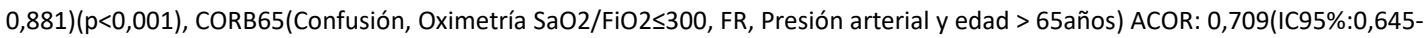
$0,773)(p<0,001)$.

\section{CONCLUSIONES}

Se encontró un buen rendimiento diagnóstico para un puntaje compuesto con valores de saturación de oxígeno similar al CURB-65 y CRB-65 para la predicción de desenlaces de mortalidad y requerimiento de ventilación mecánica en pacientes con neumonía. La relación $\mathrm{SaO} 2 / \mathrm{FiO} 2$ tiene un mayor rendimiento pronóstico que los valores de $\mathrm{SaO} 2$ en estos pacientes. Adjuntos:COR-Neumonia.docx

COR-65 (Confusión, Oxigenación SaO2/FiO2 300 , FR, PA) como Puntaje Alternativo para la Predicción de Complicaciones en Neumonía.

Bastidas Goyes Alirio, Sharon Lechtig, Oscar Arcos, Victor Ramirez, Daniela Granados.

Universidad de La Sabana, Clínica Universidad de La Sabana - Chía, Colombia.

Introducción: La medición de la oxigenación en pacientes con neumonía está integrada en los puntajes pronósticos de PSI/PORT, SCAP, SOAR, ATS, IDSA y SMART-COP, sin embargo, no se conoce el rendimiento de un puntaje que integre valores de saturación arterial de oxígeno/fracción inspirada de oxígeno (SaO2/FiO2) para desenlaces de mortalidad y ventilación mecánica (VM) en estos pacientes. El objetivo de este estudio es determinar la validez pronostica de un puntaje que integre la relación SaO2/FiO2 en neumonía.

Métodos: Estudio de cohorte retrospectiva, en sujetos mayores de 18 años que se ingresaron al servicio de urgencias y hospitalización de dos centros de atención en salud con diagnóstico de neumonía adquirida en la comunidad, se calculó el puntaje de CURB-65, CRB-65 al ingreso, y toma de gases arteriales, neumonía se definió como un cuadro de infección del parénquima pulmonar aguda, al menos un signo de respuesta inflamatoria sistémica, asociada con una opacidad nueva en la radiografía de tórax. Se evalúo posteriormente el rendimiento pronóstico de la $\mathrm{SaO} 2$ y de la relación $\mathrm{SaO} 2 / \mathrm{FiO} 2$ de ingreso con fallecimiento y requerimiento de VM, posteriormente con un punto de corte $\leq 300$ de SaO2/FiO2 se evaluó un puntaje compuesto con esta variable y las variables habituales del CURB.

Resultados: Ingresaron al análisis 487 sujetos, el 6,4\% falleció, 18,5\% requirió VM, 18,3\% con ingreso a UCl, el promedio de edad fue de 69,8(DE:20,8) años, 103(62,2\%) de sexo masculino, el promedio de FR fue: 22,1(DE:4,7), PAS:123,0(DE:23,5), PAD: 71,7(DE:14,5), BUN: 26,0(DE:17,0), SaO2:88,1(DE:6,8), para muerte el CURB-65 mostró un ACOR de: 0,685 (IC95\%:0,594-

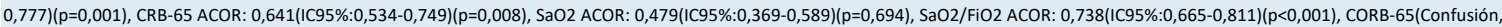
Oximetría SaO2/FiO2 $\leq 300$, FR, Presión arterial y edad > 65años) ACOR: 0,710(IC95\%:0,611-0,809)(p<0,001), para VM el CURB-65 mostró un ACOR de: 0,612(0,543-0,681)(p=0,001), CRB-65 ACOR:

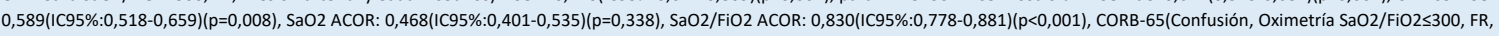
Presión arterial y edad > 65años) ACOR: 0,709(IC95\%:0,645-0,773)(p<0,001).

Conclusión: Se encontró un buen rendimiento diagnóstico para un puntaje compuesto con valores de saturación de oxígeno similar al CURB-65 y CRB-65 para la predicción de desenlaces de mortalidad y requerimiento de ventilación mecánica en pacientes con neumonía. La relación SaO2/FiO2 tiene un mayor rendimiento pronóstico que los valores de SaO2 en estos pacientes. 